\title{
Sobrevida em hemodiálise crônica: estudo de uma coorte de 1.009 pacientes em 25 anos
}

\author{
Survival in chronic hemodialysis: study of a cohort of 1,009 \\ patients in 25 years
}

\begin{abstract}
Autores
Luiz Alberto Michet da

Silva ${ }^{1,2}$

Nereu Francisco

Mezzomo ${ }^{1,2}$

Henry Mor Pansard ${ }^{1,2}$

Luiz Cláudio Arantes ${ }^{1,2}$

Werner Rempel ${ }^{1,4}$

Laércio Cassol

Argenta ${ }^{1,2}$

Arnaldo Teixeira

Rodrigues $^{1,2}$

Rafael Lampert

Cauduro ${ }^{1,3}$

Daniel Melchiades da

Silva ${ }^{1,3}$

Clóvis Luís Konopka ${ }^{2,4}$

Elton Luíz Weber ${ }^{2,4}$

Geni Burg ${ }^{1}$

Clara Maria Trevisan ${ }^{1,2}$

Macilene Regina

Pauletto 1,2

Odete Teresinha

Portela ${ }^{1}$

Onélia Costa

Cordenuzzi ${ }^{1}$

Juliana Peres Rist ${ }^{1}$

Arlete Maria Timm ${ }^{1,2}$

Carina Vendrusculo

Mezomo ${ }^{1}$

Priscila Kanheski

Moreira $^{2}$

Ana Lucia Santos ${ }^{2}$

${ }^{1}$ Clínica Renal de Santa Maria, Santa Maria

${ }^{2}$ Hospital Universitário

de Santa Maria

${ }^{3} \mathrm{Hospital}$ de Caridade

Dr. Astrogildo de

Azevedo, Santa Maria

${ }^{4}$ Hospital Municipal Casa

de Saúde, Santa Maria

Data de submissão: 28/05/2009

Data de aprovação: 12/08/2009

Correspondência para:

Luiz Alberto Michet da Silva

Rua Carlos Brenner, 100

Santa Maria - RS

\section{Resumo}

Introdução: A maioria dos pacientes com doença renal crônica terminal depende de hemodiálise (HD) para a manutenção de sua vida. A análise dos fatores que influenciam na sobrevida pode auxiliar na busca contínua por melhores resultados. Métodos: Analisamos 1.009 pacientes tratados por HD crônica em três unidades de diálise de Santa Maria, RS, Brasil, durante 25 anos (1982-2007). Resultados: A sobrevida (método de Kaplan-Meier) em 1, 2 e 5 anos foi de $91 \%$, $84 \%$ e $64 \%$, respectivamente. No modelo proporcional de Cox, tiveram influência estatisticamente significativa sobre o risco de mortalidade: idade ao iniciar HD (aumento de 4,5\% por ano a mais; $\mathrm{p}=0,0001)$, presença de diabetes (aumento de $56 \% ; \mathrm{p}=0,001$ ) e ano de início da HD (redução de $5,2 \%$ por ano mais tarde; $p=0,0001$ ). A sobrevida foi significativamente melhor para pacientes que iniciaram HD de 1997 a 2007 do que para os que iniciaram de 1982 a 1996, tanto em diabéticos $(54 \%$ versus $41 \%$ em 5 anos; $\mathrm{p}=0,01$ ) como não diabéticos ( $72 \%$ versus $65 \%$ em 5 anos; $p=0,045)$, embora, nestes, a idade tenha sido significativamente maior no período mais recente. Conclusões: A presença de diabete e cada ano a mais na idade determinaram risco significativamente aumentado. Cada ano subsequente do calendário trouxe um risco significativamente menor. Nos anos mais recentes, a melhora de sobrevida foi maior para pacientes diabéticos e idosos, sendo atribuída a avanços diagnósticos e terapêuticos e melhor qualidade global do programa dialítico.
\end{abstract}

CEP: $97050-100$

Tel: (55) 3221-6496 /

(55) 3027-3394 / (55) 9964-6221

Declaramos a inexistência de conflitos de interesse.
Palavras-chave: doença renal crônica terminal, hemodiálise, sobrevida.

[J Bras Nefrol 2009;31(3):190-197]CElsevier Editora Ltda.

\section{Abstract}

Introduction: Most patients with endstage renal disease depend on hemodialysis (HD) for life maintenance. Analysis of factors influencing survival can assist in the continuous search for better results. Methods: We analysed 1,009 patients treated with chronic HD in three dialysis units in the city of Santa Maria, RS, Brazil, for 25 years (1982-2007). Results: Survival (Kaplan-Meier method) at 1,2, and 5 years was $91 \%, 84 \%$, and $64 \%$, respectively. In Cox regression model, the variables with a statistically significant impact on mortality risk were: age at starting HD (increase of $4.5 \%$ per additional year; $p$ $=0.0001$ ), presence of diabetes (increase of $56 \% ; \mathrm{p}=0.001$ ), and year of beginning HD treatment (reduction of $5.2 \%$ for each subsequent year; $p=0.0001$ ). Survival was significantly better for patients who started HD from 1997 to 2007 than for those who started from 1982 to 1996 , for both diabetic (54\% vs. $41 \%$ at 5 years; $\mathrm{p}=0.01)$ and nondiabetic patients $(72 \%$ vs. $65 \%$ at 5 years; $p=0.045)$, although nondiabetic patients were significantly older in the latter period. Conclusions: The presence of diabetes and each additional year in age led to significantly increased risk. Regarding the year of the beginning of the HD program, a significantly smaller risk was observed for each subsequent calendar year. The increase in survival achieved in more recent years was greater for diabetic and older patients. It was attributed to diagnostic and therapeutic improvements and better overall quality of the dialysis program.

Keywords: end-stage renal failure, hemodialysis, survival. 


\section{INTRODUÇÃO}

O número de pacientes com Doença Renal Crônica Terminal ou Estágio 5 (DRC 5) tem aumentado constantemente em todo o mundo. ${ }^{1-6} \mathrm{~A}$ terapia renal substitutiva por meio de diálise e transplante renal tem obtido bons resultados, mas implica altos custos, com substancial impacto nos orçamentos de saúde pública e nos programas de assistência à saúde de muitas instituições em vários países. ${ }^{1,7-14}$ Embora um transplante renal bem-sucedido seja reconhecidamente a melhor opção de tratamento, ${ }^{15-19}$ o número de transplantes realizado é muito inferior ao número de novos casos de DRC 5 / milhão de habitantes / ano, mesmo nos países com o melhor desempenho em captação de órgãos e realização de transplantes. ${ }^{20-22}$ Desse modo, a maioria dos pacientes com DRC 5 depende de diálise crônica para a manutenção de sua vida, e hemodiálise (HD) é a principal forma de tratamento utilizada por eles. Neste trabalho, analisamos uma coorte de 1.009 pacientes com DRC 5 em programa de hemodiálise crônica tratados em um único centro durante um período de 25 anos, com o objetivo de conhecer a sobrevida obtida e a influência de diversas variáveis demográficas, clínicas e técnicas nesse desfecho.

\section{Métodos}

Analisaram-se todos os pacientes adultos com DRC $5(\mathrm{n}=1037)$ que iniciaram programa regular de HD crônica entre 3 de maio de 1982 e 3 de maio de 2007 na cidade de Santa Maria, RS, Brasil. Não foi possível obter informações adequadas de 28 pacientes $(2,7 \%)$, que não foram incluídos. Três unidades de HD (Unidade de Hemodiálise da Clínica Renal de Santa Maria no Hospital Municipal Casa de Saúde, Unidade de Hemodiálise da Clínica Renal de Santa Maria no Hospital de Caridade Dr. Astrogildo de Azevedo e Unidade de Hemodiálise do Serviço de Nefrologia no Hospital Universitário de Santa Maria) ofereceram tratamento para os 1.009 pacientes da coorte estudada. A data estabelecida como o final do período de seguimento para o estudo foi 22 de outubro de 2007.

\section{HEMODIÁLISE}

De 1982 até 1986, foram utilizados dialisadores tipo coil e máquinas com geometria "RSP" (recirculating single pass), em que um tanque de 120 litros continha a solução de diálise preparada para uma sessão de 4 horas. A partir de 1987, as três unidades de hemodiálise passaram a utilizar dialisadores de fibras ocas capilares e máquinas com preparação central da solução de diálise, distribuída para módulos individuais. Máquinas proporcionadoras passaram a ser utilizadas para todos os pacientes a partir de 1997. O tratamento da água incluiu filtros de sedimentação (areia) e tanques de carvão ativado mais deionizadores de 1982 até 1994 e osmose reversa de 1995 em diante. Dialisador mais linha arterial e venosa foram reutilizados para o mesmo paciente como procedimento de rotina, de acordo com as técnicas descritas..$^{23}$ Dialisadores reutilizados eram descartados sempre que seu volume de "priming" se tornasse inferior a $80 \%$ em relação ao valor inicial. O número médio de sessões realizadas com cada dialisador foi de $10 \pm 1$. Cada paciente realizou três sessões de HD por semana. Quando necessário, sessões adicionais eram realizadas por razões específicas, principalmente hipervolemia e hiperpotassemia. A duração de cada sessão foi de 4 horas para 858 pacientes ( $85 \%), 4,3$ horas para 81 pacientes $(8 \%)$ e 3,5 horas para 70 pacientes $(7 \%)$. O método para se adequar a diálise de 1982 até 1996 consistiu em avaliação clínica e exames laboratoriais mensais (ureia, creatinina, potássio, cálcio, fósforo, bicarbonato, hematócrito, hemoglobina, proteínas totais, albumina). De 1997 até 1999, adicionou-se avaliação mensal da taxa de redução da ureia em uma sessão como marcador de adequação, buscando-se valor igual ou superior a 65\%. De 2000 até 2007 , a avaliação mensal do KT/V por um programa computadorizado foi utilizada como ferramenta para auxiliar na adequação da diálise (objetivo de 1.2 ou mais), juntamente com avaliação clínica.

Em qualquer uma das três unidades de HD, além da equipe de enfermagem, composta por enfermeira, auxiliares e técnicos de enfermagem, havia sempre um nefrologista, desde o início até o final de cada sessão.

\section{ColetA DE DADOS}

Foi feita análise retrospectiva nos prontuários das unidades de $\mathrm{HD}$ e nos prontuários hospitalares de cada paciente, colhendo-se informações desde 1982; também foram obtidos dados dos sistemas de registro computadorizado das unidades de hemodiálise, disponíveis desde 1998. Registrou-se para cada paciente: idade ao iniciar o programa de HD, sexo, raça, doença causadora da insuficiência renal, datas inicial e final do tratamento hemodialítico e motivo de saída do programa de HD. A data inicial correspondeu ao real início do programa de $\mathrm{HD}$, sem intervalo de tempo entre o começo do programa de diálise e a inclusão no estudo. O censo dos casos para análise da sobrevida incluiu o tempo desde o início do programa de HD até a ocorrência de morte por qualquer causa ou a saída do 
programa por recuperação de função renal, transplante renal, transferência para diálise peritoneal, transferência para outro centro, abandono do tratamento ou até a data final do período de seguimento do estudo.

\section{Análise estatística}

A sobrevida dos pacientes foi determinada pelo método de Kaplan-Meier; o Teste Log Rank foi utilizado para comparação de curvas de sobrevida. Análise multivariada por meio de Regressão de Cox foi utilizada para estudar a influência de diversas variáveis clínicas e demográficas na sobrevida dos pacientes. A comparação entre dois grupos foi realizada pelo Teste $t$ para variáveis contínuas e pelo Teste do QuiQuadrado para variáveis categóricas. Todas as análises estatísticas foram desenvolvidas usando o SPSS para Windows (versão 11.0, Chicago, Il, USA).

\section{Resultados}

Dos 1.009 pacientes, $55 \%$ eram homens; $84 \%$, caucasianos; e 16\%, afro-americanos. A idade para o grupo todo foi de $51 \pm 16$ anos

A doença de base foi atribuída a nefropatia diabética em 242 casos $(24 \%)$, nefroesclerose hipertensiva em 172 casos (17\%), glomerulonefrite crônica em 151 casos $(15 \%)$, doença renal policística em 52 casos $(5 \%)$, pielonefrite crônica em 41 casos ( $4 \%)$, outra em 121 casos (12\%) e desconhecida em 230 casos $(23 \%)$. A depuração da creatinina endógena imediatamente antes do início do programa de hemodiálise foi entre 5 e $12 \mathrm{~mL} / \mathrm{min} / 1.73 \mathrm{~m}^{2}$ para não diabéticos e entre 10 e $15 \mathrm{~mL} / \mathrm{min} / 1.73 \mathrm{~m}^{2}$ para pacientes diabéticos.

O acesso vascular foi fístula arteriovenosa em 948 pacientes $(94 \%)$ e prótese vascular em 61 pacientes $(6 \%)$. O local do acesso vascular foi o antebraço ou o braço em 962 pacientes (95\%) e a coxa em 47 pacientes $(5 \%)$. Acesso por meio de cateter venoso central de duplo lúmen foi utilizado temporariamente para pacientes que ainda não apresentavam fístula arteriovenosa no momento em que necessitaram iniciar hemodiálise ou no período de falência de uma fístula previamente funcionante, até se dispor de uma nova em condições de uso.

Para a coorte total, a sobrevida dos pacientes foi de $91 \%$ em 1 ano (750 pacientes em risco), $84 \%$ em 2 anos ( 579 pacientes em risco), $78 \%$ em 3 anos (433 pacientes em risco), 64\% em 5 anos (250 pacientes em risco), $53 \%$ em 7 anos (143 pacientes em risco), $51 \%$ em 8 anos ( 114 pacientes em risco) e $41 \%$ em 10 anos (66 pacientes em risco).
Considerando a evolução final dos 1.009 pacientes, 16 recuperaram função renal $(1,6 \%), 140$ foram transplantados $(14 \%), 68$ foram transferidos para diálise peritoneal $(6,7 \%), 199$ foram transferidos para outro centro $(19,7 \%), 7$ abandonaram o tratamento $(0,7 \%)$, 250 estavam recebendo HD regular no final do período de estudo (24,8\%) e 329 foram a óbito (32,5\%).

Análise univariada mostrou sobrevida significativamente inferior para pacientes diabéticos do que para não diabéticos $(\log \operatorname{Rank}=30,43 ; \mathrm{p}=0,00001$; Figura 1). A idade dos pacientes diabéticos $(58 \pm 12$ anos) foi significativamente mais elevada do que a dos não diabéticos $(49 \pm 16$ anos $)(\mathrm{p}=0,0001)$. A sobrevida respectivamente para não diabéticos e diabéticos foi de $92 \%$ versus $87 \%$ em 1 ano, $87 \%$ versus $77 \%$ em 2 anos e $69 \%$ versus $50 \%$ em 5 anos.

Por outro lado, análise univariada não mostrou diferença estatisticamente significativa de sobrevida entre homens e mulheres $(\log \operatorname{Rank}=2,58 ; \mathrm{p}=0,108)$.

A Tabela 1 mostra os resultados da Regressão de Cox analisando o impacto de variáveis clínicas e demográficas na sobrevida dos pacientes. As variáveis que mostraram influência estatisticamente significativa na sobrevida foram: idade ao iniciar HD, sexo, presença de diabetes e ano de início da HD. Houve um "hazard ratio" (HR) significativamente aumentado para cada ano a mais na idade ao iniciar HD e para a presença de diabetes. Por outro lado, considerando-se o ano de início do programa de HD, observou-se um HR

Figura 1. Sobrevida de pacientes diabéticos vs. não diabéticos $(p=0,00001)$.

$\begin{array}{lccc}\text { Pacientes em risco: } & 1 \text { ano } & 2 \text { anos } & 5 \text { anos } \\ \text { Não diabéticos: } & 579 & 455 & 207 \\ \text { Diabéticos: } & 171 & 124 & 43\end{array}$

Sobrevida cumulativa

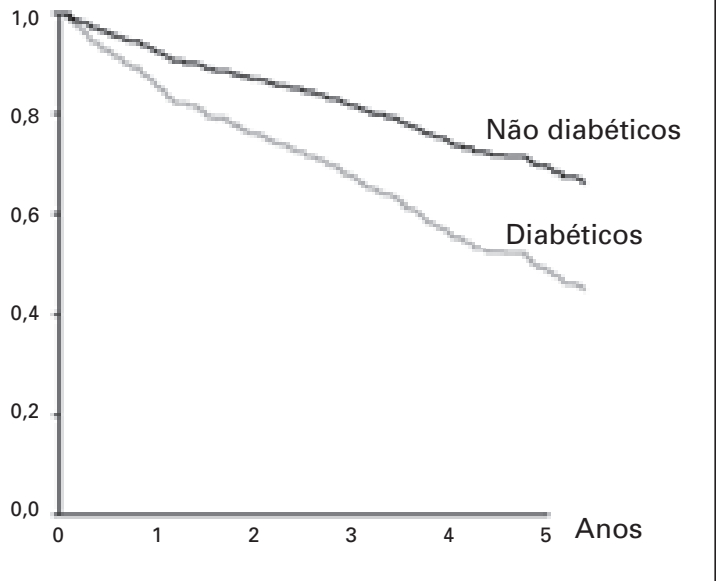




\begin{tabular}{|c|c|c|c|c|}
\hline Tabela 1 & \multicolumn{4}{|c|}{$\begin{array}{l}\text { INFLUÊNCIA DE DIVERSAS VARIÁVEIS NA SOBREVIDA DOS PACIENTES } \\
\text { (Modelo PROPORCIONAL - REgRESSÃo DE CoX) } \quad \text { N = 1.009; Período: } 1982 \text { - } 2007\end{array}$} \\
\hline \multicolumn{2}{|l|}{ Variável } & Hazard Ratio & IC $95 \%$ & $\mathrm{p}$ \\
\hline \multicolumn{2}{|c|}{ Idade (anos) } & 1,045 & $1,036-1,054$ & 0,0001 \\
\hline \multicolumn{2}{|c|}{ Sexo (mulheres/homens) } & 1,252 & $1,002-1,564$ & 0,048 \\
\hline \multicolumn{2}{|c|}{ Raça } & 0,889 & $0,655-1,206$ & 0,449 \\
\hline \multicolumn{2}{|c|}{ Presença de Diabetes } & 1,556 & $1,208-2,005$ & 0,001 \\
\hline \multicolumn{2}{|c|}{ Ano de início da Hemodiálise* } & 0,948 & $0,931-0,966$ & 0,0001 \\
\hline
\end{tabular}

* Ano do calendário em que cada paciente iniciou HD (de 1982 a 2007)

Desempenho Global do Modelo: (Qui-Quadrado $=141,403 ; p=0,0001$ )

significativamente menor para cada ano subsequente do calendário. No conjunto de variáveis analisadas, o fator sexo mostrou um efeito marginalmente significativo, com sobrevida levemente superior no sexo masculino. A variável raça não teve impacto estatisticamente significativo na sobrevida dos pacientes.

Como a análise dos dados indicou um efeito estatisticamente significativo do ano em que cada paciente iniciou hemodiálise, com melhora progressiva de sobrevida em cada ano subsequente, decidiu-se estudar comparativamente dois grupos de pacientes, respectivamente de um período prévio e de outro posterior à disponibilidade de recursos técnicos e medicamentosos que presumivelmente deveriam produzir melhoria na qualidade do programa dialítico. $\mathrm{O}$ período 1 incluiu os pacientes que iniciaram HD de 1982 a $1996(n=422)$ e o período 2, os que iniciaram HD de 1997 a 2007 ( $\mathrm{n}=$ 587). Considerouse 1996-1997 como transição por ter correspondido à época de implementação do seguinte conjunto de itens: tratamento da água por osmose reversa; uso de máquinas proporcionadoras; uso regular de eritropoetina recombinante humana; e mensuração rotineira da adequação da diálise.

Os valores médios de hematócrito (\%) e hemoglobina $(\mathrm{g} / \mathrm{dL})$ antes e depois do uso regular de eritropoetina recombinante humana foram, respectivamente, $24 \pm 4$ versus $33 \pm 5(\mathrm{p}=0.0001)$ e $7,3 \pm 2,4$ versus $10,5 \pm$ $2,1(\mathrm{p}=0.0001)$.

Conforme se observa na Tabela 2, os pacientes do período 2 apresentaram idade significativamente mais avançada, uma proporção significativamente maior de diabéticos e uma proporção significativamente menor de afro-americanos.

A sobrevida no período 2 foi significativamente superior em relação à sobrevida no período $1(\log$ Rank $=5,8 ; \mathrm{p}=0,016)$, correspondendo a $93 \%$ versus $87 \%$ em 1 ano, $89 \%$ versus $78 \%$ em 2 anos e $67 \%$ versus $60 \%$ em 5 anos. A comparação das curvas de sobrevida dos dois períodos é mostrada na Figura 2.

Estudou-se separadamente o que ocorreu em pacientes diabéticos e não diabéticos, comparando-se o período 1 e o período 2 .

Nos pacientes não diabéticos, a sobrevida respectivamente nos períodos 2 e 1 foi de $94 \%$ versus $90 \%$ em 1 ano, $90 \%$ versus $83 \%$ em 2 anos e $72 \%$ versus $65 \%$ em 5 anos $(\log \operatorname{Rank}=4,02 ; \mathrm{p}=0,045)$. As curvas correspondentes são mostradas na Figura 3. Os pacientes não diabéticos do período 2 tiveram idade significativamente mais elevada (52 \pm 16 anos) do que os do período 1 (46 \pm 16 anos) ( $\mathrm{p}=0,0001$ ).

Nos pacientes diabéticos, a sobrevida, respectivamente, nos períodos 2 e 1 foi de $93 \%$ versus $75 \%$ em 1 ano, $86 \%$ versus $59 \%$ em 2 anos e $54 \%$ versus $41 \%$

\begin{tabular}{|c|c|c|c|c|c|}
\hline \multirow[t]{2}{*}{ Tabela 2} & \multicolumn{5}{|c|}{ 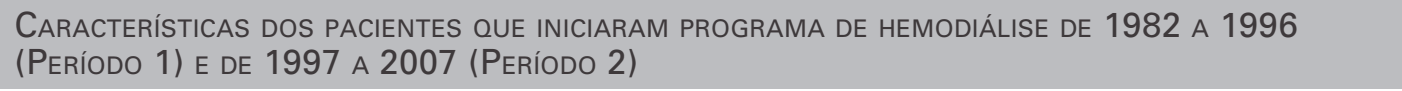 } \\
\hline & & Período 1 (1982-1996) & Período 2 ( & 997-2007) & $\mathrm{p}$ \\
\hline \multicolumn{2}{|l|}{$\mathrm{N}$} & 422 & \multicolumn{2}{|c|}{587} & \\
\hline \multicolumn{2}{|c|}{ Idade (anos) } & $48 \pm 16$ & \multicolumn{2}{|c|}{$54 \pm 15$} & 0,0001 \\
\hline \multicolumn{2}{|c|}{ Diabéticos } & $78 / 422 \quad(19 \%)$ & 164 / 587 & $(28 \%)$ & 0,0001 \\
\hline \multicolumn{2}{|c|}{ Sexo (mulheres) } & $177 / 422 \quad(42 \%)$ & $276 / 587$ & $(47 \%)$ & 0,124 \\
\hline \multicolumn{2}{|c|}{ Raça (afro-americanos) } & $84 / 422 \quad(20 \%)$ & 82 / 587 & $(14 \%)$ & 0,009 \\
\hline
\end{tabular}


Figura 2. Sobrevida no período $1 \mathrm{vs}$. período $2(\mathrm{p}=0,016)$.

$\begin{array}{cccc}\text { Pacientes em risco: } & 1 \text { ano } & 2 \text { anos } & 5 \text { anos } \\ \text { Período 1: } & 310 & 232 & 133 \\ \text { Período 2: } & 440 & 347 & 117\end{array}$

Sobrevida cumulativa

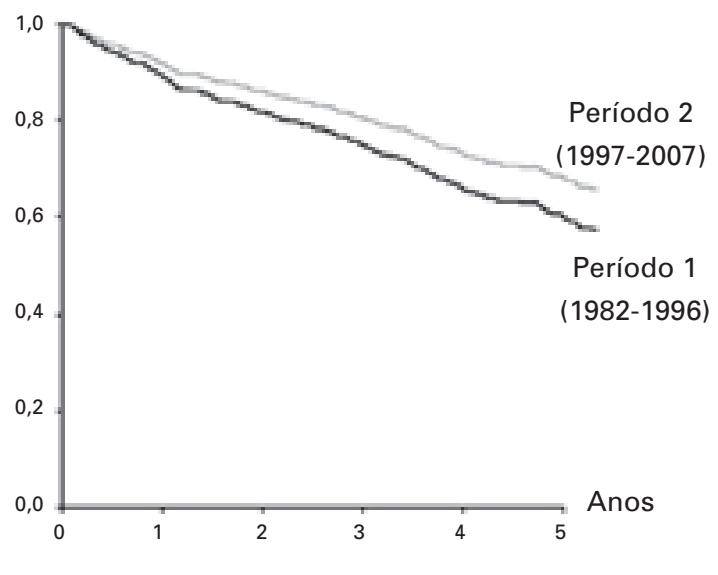

Figura 3. Sobrevida de não diabéticos no período 1 vs. período $2(p=0,045)$.

$\begin{array}{cccc}\text { Pacientes em risco: } & 1 \text { ano } & 2 \text { anos } & 5 \text { anos } \\ \text { Período 1: } & 262 & 199 & 116 \\ \text { Período 2: } & 317 & 256 & 91\end{array}$

Sobrevida cumulativa

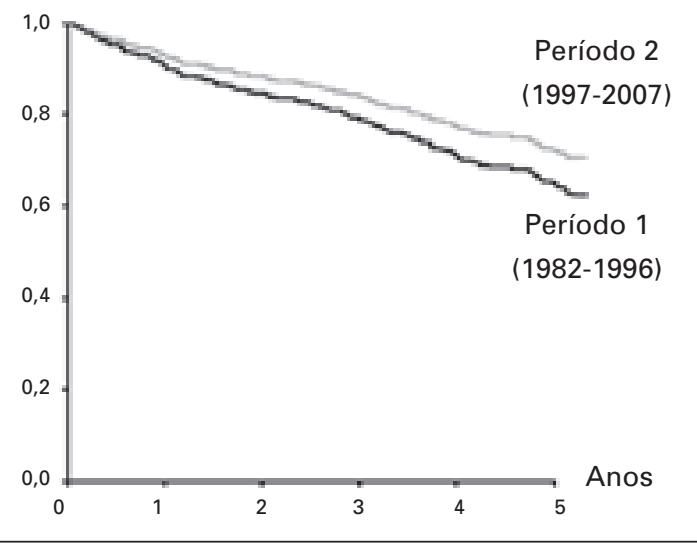

em 5 anos $(\log \operatorname{Rank}=6,629 ; \mathrm{p}=0,01)$, o que corresponde às curvas mostradas na Figura 4. Não houve diferença estatisticamente significativa entre a idade dos pacientes diabéticos do período 2 (58 \pm 12 anos) e do período 1 (57 \pm 12 anos) ( $\mathrm{p}=0,369)$.

\section{Dıscussão}

Análise de sobrevida tem sido uma ferramenta de grande valor para avaliar os resultados de programas
Figura 4. Sobrevida de diabéticos no período 1 vs. período 2 ( $p=0,01$ ).

$\begin{array}{cccc}\text { Pacientes em risco: } & 1 \text { ano } & 2 \text { anos } & 5 \text { anos } \\ \text { Período 1: } & 48 & 32 & 17 \\ \text { Período 2: } & 123 & 92 & 26\end{array}$

Sobrevida cumulativa

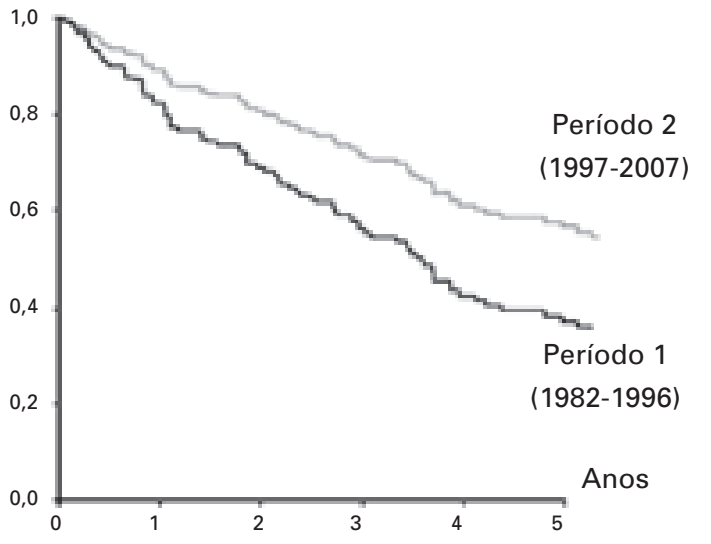

de HD crônica. ${ }^{1,24-29} \mathrm{O}$ estudo dos dados de cada centro é útil para analisar a evolução dos pacientes e para verificar os resultados obtidos por essa modalidade de tratamento de alto custo. Estudos com grandes séries de pacientes mostram taxas de sobrevida de $88 \%$ em 1 ano, $77 \%$ em 2 anos e $50 \%$ em 5 anos nos países da Associação Europeia de Diálise e Transplante ${ }^{24} \mathrm{e}$ $79 \%$ em 1 ano, $61 \%$ em 2 anos e $36 \%$ em 5 anos nos Estados Unidos. ${ }^{1}$ Sobrevida de $90 \%$ em 1 ano, $73 \%$ em 5 anos e $54 \%$ em 10 anos associada com HD lenta prolongada tem sido publicada pelo grupo de Tassin, na França. ${ }^{29}$

$\mathrm{Na}$ coorte aqui estudada, a sobrevida foi de $91 \%$ em 1 ano, $84 \%$ em 2 anos, $64 \%$ em 5 anos, $51 \%$ em 8 anos e $41 \%$ em 10 anos.

O presente trabalho mostrou impacto estatisticamente significativo da idade do paciente na sobrevida, constatando-se um HR aumentado para cada ano a mais na idade ao iniciar o programa de HD. Um efeito semelhante foi também detectado por outros pesquisadores. ${ }^{1,24}$

Pacientes diabéticos tiveram sobrevida significativamente inferior aos não diabéticos, o que também tem sido encontrado em diversos outros estudos. ${ }^{30-32}$

Para cada ano subsequente do calendário em que os pacientes iniciavam programa de $\mathrm{HD}$, houve um HR significativamente menor, o que deve ser atribuído 
a diversas melhorias relacionadas a equipamentos, técnicas e medicamentos durante esse período de 25 anos. Isso incluiu melhor tratamento da água, melhores máquinas de hemodiálise e dialisadores, melhores maneiras de atingir e medir adequação da diálise e melhor controle da anemia por meio de eritropoetina recombinante humana. O impacto de uma dessas variáveis, especificamente níveis mais elevados de hematócrito e hemoglobina, diminuindo mortalidade e número de hospitalizações, tem sido demonstrado por outros pesquisadores, em estudos com grande número de pacientes. ${ }^{33}$ Por outro lado, durante o período do presente estudo, ocorreram também diversos outros melhoramentos diagnósticos e terapêuticos além do tratamento dialítico em si, como maior disponibilidade de procedimentos para doença cardiovascular, incluindo angioplastias, implantes de stents e cirurgias de revascularização, o que certamente também contribuiu para a obtenção de melhores resultados.

A comparação da evolução dos pacientes que iniciaram HD no período 1 (1982-1996) com os que iniciaram no período 2 (1997-2007) mostrou que os do período mais recente tiveram sobrevida significativamente melhor, apesar de idade mais avançada e de uma proporção significativamente maior de diabéticos. Tal fato sugere a importância do impacto de melhor qualidade da diálise, proporcionando melhor sobrevida, mesmo para grupos que incluem maior proporção de pacientes com risco aumentado, como idosos e diabéticos.

A análise separada de não diabéticos e diabéticos evidenciou que, em ambos os casos, houve melhora significativa de sobrevida do período 1 para o período 2. Entretanto, o efeito foi nitidamente mais pronunciado no grupo de diabéticos, indicando que tais pacientes tiveram um benefício maior com as melhorias diagnósticas e terapêuticas dos anos mais recentes.

Embora a duração ideal das sessões de hemodiálise permaneça incerta, sabe-se que pacientes submetidos a sessões mais curtas ficam frequentemente com sobrecarga hidrossalina, hipertensão não controlada, hipertrofia ventricular esquerda, disfunção diastólica e alta mortalidade cardiovascular, ${ }^{34,35}$ enquanto sessões mais longas e ultrafiltração mais lenta são geralmente associadas com menor morbidade cardiovascular e melhor sobrevida. ${ }^{29,36,37}$ Um estudo comparativo mostrou que unidades de HD na Europa e no Japão tinham tempo médio de duração da sessão e KT/V significativamente maior do que as unidades dos Estados Unidos; o mesmo estudo também demonstrou que sessões mais prolongadas eram associadas com menor mortalidade, e, considerando as três re- giões citadas, as unidades de HD do Japão mostravam os maiores tempos de duração das sessões e o menor risco de mortalidade. ${ }^{36}$

No presente estudo, a duração das sessões foi de 4 horas ou mais para $93 \%$ dos pacientes; é possível que este tempo consideravelmente longo de diálise tenha sido um dos fatores com influência positiva na sobrevida.

A reutilização de dialisadores tem sido objeto de controvérsia há muito tempo; trata-se de uma prática rotineira em muitos centros, enquanto outros descartam cada dialisador após uso único. ${ }^{38-40}$ Os resultados deste trabalho sugerem que o reuso em si, se adequadamente e cuidadosamente realizado, pode ser perfeitamente compatível com um programa de hemodiálise de adequada qualidade. Além disso, a reutilização diminui custos e reduz a quantidade de material plástico descartado, o que pode contribuir para diminuir o impacto ambiental a curto e longo prazo.

$\mathrm{O}$ atendimento nefrológico médico e de enfermagem não foi modificado ao longo do período deste estudo, de modo que cada paciente foi sempre avaliado em cada sessão de HD, três vezes por semana, com o objetivo de detectar e tratar precocemente novas condições, como complicações infecciosas ou cardiovasculares. Desse modo, o aumento de sobrevida obtido no período mais recente não foi devido a nenhuma mudança de ação dos profissionais da equipe, devendo ser atribuído às melhorias técnicas, diagnósticas e terapêuticas já descritas. Outros autores têm também descrito o impacto positivo que uma melhor qualidade do cuidado oferecido tem nos resultados obtidos. ${ }^{25,26,29,36}$

Este trabalho tem a limitação inerente a todas as pesquisas retrospectivas; 28 casos tiveram de ser excluídos por inexistência de informações adequadas. Entretanto, em todos os casos incluídos, foi possível obter informações definidas sobre as variáveis analisadas. A amostra estudada representou 97,3\% dos pacientes com DRC 5 que iniciaram programa regular de HD durante o período considerado, sugerindo que seja uma amostra representativa da população adulta com doença renal terminal que necessitou de tratamento hemodialítico crônico.

Em conclusão, este estudo mostrou sobrevida significativamente inferior para pacientes diabéticos do que para não diabéticos, um risco significativamente maior para cada ano a mais na idade ao iniciar o programa de HD e um risco significativamente menor a cada ano subsequente do calendário em que os pacientes iniciaram tratamento dialítico, não havendo influência estatisticamente significativa de sexo e raça na sobrevida. $\mathrm{O}$ estudo ainda permitiu concluir que 
houve impacto positivo de melhorias diagnósticas, terapêuticas e da qualidade global do programa dialítico oferecido nos anos mais recentes. Essa melhor sobrevida ocorreu apesar de os pacientes recentes serem mais idosos e foi muito mais pronunciada nos pacientes diabéticos. Tais dados indicam que é fundamental a busca de melhora contínua de todos os itens do tratamento dialítico, o que beneficia todos os pacientes, mas especialmente os de maior risco, como idosos e diabéticos, que representam parcela cada vez maior dos que entram em tratamento.

\section{Agradecimento (In memoriam)}

Ao Dr. Carlos da Cunha Cauduro, primeiro professor de Nefrologia do curso de Medicina da Universidade Federal de Santa Maria e primeiro nefrologista da Clínica Renal de Santa Maria, cuja humanidade, conhecimento científico e habilidade clínica serão sempre uma forte inspiração para todos os que tiveram o privilégio de trabalhar com ele.

\section{REFERÊNCIAS}

1. U.S. Renal Data System, USRDS 2008 Annual Data Report: Atlas of Chronic Kidney Disease and End-Stage Renal Disease in the United States, National Institutes of Health, National Institute of Diabetes and Digestive and Kidney Diseases, Bethesda, MD, 2008.

2. Ryan TP, Sloand JA, Winters PC, Corsetti JP, Fisher SG. Chronic kidney disease prevalence and rate of diagnosis. Am J Med 2007; 120:981-6.

3. Imai E, Yamagata K, Iseki K et al. Kidney disease screening program in Japan: history, outcome, and perspectives. Clin J Am Soc Nephrol 2007; 2:1360-6.

4. Xie Y, Chen X. Epidemiology, major outcomes, risk factors, prevention and management of chronic kidney disease in China. Am J Nephrol 2008; 28:1-7.

5. Dirks J. A world perspective on renal care: the challenges of prevention and treatment. EDTNA ERCA J 2005; 31:72-4.

6. Schieppati A, Remuzzi G. Chronic renal diseases as a public health problem: epidemiology, social, and economic implications. Kidney Int Suppl 200:S7-S10.

7. Kleophas W, Reichel H. International study of health care organization and financing: development of renal replacement therapy in Germany. Int J Health Care Finance Econ 2007; 7:185-200.

8. Durand-Zaleski I, Combe C, Lang P. International Study of Health Care Organization and Financing for end-stage renal disease in France. Int J Health Care Finance Econ 2007; 7:171-83.

9. Luño J. The organization and financing of end-stage renal disease in Spain. Int J Health Care Finance Econ 2007; 7:253-67.

10. Van Biesen W, Lameire N, Peeters P, Vanholder R. Belgium's mixed private/public health care system and its impact on the cost of end-stage renal disease. Int J Health Care Finance Econ 2007; 7:133-48.
11. Pontoriero G, Pozzoni P, Vecchio LD, Locatelli F. International Study of Health Care Organization and Financing for renal replacement therapy in Italy: an evolving reality. Int J Health Care Finance Econ 2007; 7:20115 .

12. Nicholson T, Roderick P. International Study of Health Care Organization and Financing of renal services in England and Wales. Int J Health Care Finance Econ 2007; 7:283-99.

13. Prodjosudjadi W. Incidence, prevalence, treatment and cost of end-stage renal disease in Indonesia. Ethn Dis 2006; 16(2 Suppl 2):S2-14-6.

14. Jha V. End-stage renal care in developing countries: the India experience. Ren Fail 2004; 26:201-8.

15. McDonald SP, Russ GR. Survival of recipients of cadaveric kidney transplants compared with those receiving dialysis treatment in Australia and New Zealand, 1991-2001. Nephrol Dial Transplant 2002; 17:2212-9.

16. Rabbat CG, Thorpe KE, Russell JD, Churchill DN. Comparison of mortality risk for dialysis patients and cadaveric first renal transplant recipients in Ontario, Canada. J Am Soc Nephrol 2000; 11:917-22.

17. Tomasz W, Piotr S. A trial of objective comparison of quality of life between chronic renal failure patients treated with hemodialysis and renal transplantation. Ann Transplant 2003; 8:47-53.

18. Oniscu GC, Brown H, Forsythe JL. How great is the survival advantage of transplantation over dialysis in elderly patients? Nephrol Dial Transplant 2004; 19:945-51.

19. Glanton CW, Kao TC, Cruess D, Agodoa LY, Abbott KC. Impact of renal transplantation on survival in end-stage renal disease patients with elevated body mass index. Kidney Int 2003; 63:647-53.

20. O’Connor KJ, Delmonico FL. Increasing the supply of kidneys for transplantation. Semin Dial 2005; 18:460-2.

21. Cohen B, Smits JM, Haase B, Persijn G, Vanrenterghem Y, Frei U. Expanding the donor pool to increase renal transplantation. Nephrol Dial Transplant 2005; 20:34-41.

22. The United Network for Organ Sharing (UNOS) and The U.S. Organ Procurement and Transplantation Network (OPTN) - http://www.optn.org.

23. Association for Advancement of Medical Information (AAMI): American National Standard. Reuse of Hemodialyzers (ANSI/AAMI RD47:2002 \& RD47:2002/ A1:2003). Arlington, VA, AAMI, 2003.

24. ERA-EDTA Registry: ERA-EDTA Registry 2006 Annual Report. Academic Medical Center, Department of Medical Informatics, Amsterdam, The Netherlands, 2008.

25. Rocco MV, Frankenfield DL, Hopson SD, McClellan WM. Relationship between clinical performance measures and outcomes among patients receiving long-term hemodialysis. Ann Intern Med 2006; 145:512-9.

26. Saudan P, Kossovsky M, Halabi G, Martin PY, Perneger TV, Western Switzerland Dialysis Study Group. Quality of care and survival of haemodialysed patients in western Switzerland. Nephrol Dial Transplant 2008; 23:1975-81.

27. Gonzalez C, Fernandez-Cean J, Gonzalez-Martinez F, Schwedt E, Mazzuchi N. Chronic dialysis in Uruguay: mortality trends from 1981 to 1998. Nephrol 2001; 21:342-8. 
28. Mazzuchi N, Schwedt E, Fernandez JM et al. Latin American Registry of dialysis and renal transplantation: 1993 annual dialysis data report. Nephrol Dial Transplant 1997; 12:2521-7.

29. Innes A, Charra B, Burden RP, Morgan AG, Laurent G. The effect of long, slow haemodialysis on patient survival. Nephrol Dial Transplant 1999; 14:919-22.

30. Villar E, Chang SH, McDonald SP. Incidences, treatments, outcomes, and sex effect on survival in patients with end-stage renal disease by diabetes status in Australia and New Zealand (1991-2005). Diabetes Care 2007; 30:3070-6.

31. Batista PB, Lopes AA, Costa FA. Association between attributed cause of end-stage renal disease and risk of death in Brazilian patients receiving renal replacement therapy. Ren Fail 2005; 27:651-6.

32. Racki S, Zaputović L, Vujicić B, Crncević-Orlić Z, Dvornik S, Mavri Z. Comparison of survival between diabetic and non-diabetic patients on maintenance hemodialysis: a single-centre experience. Diabetes Res Clin Pract 2007; 75:169-75.

33. Pisoni RL, Bragg-Gresham JL, Young EW et al. Anemia management and outcomes from 12 countries in the Dialysis Outcomes and Practice Patterns Study (DOPPS). Am J Kidney Dis 2004; 44:94-111.
34. Twardowski ZJ. Short, thrice-weekly hemodialysis is inadequate regardless of small molecule clearance. Int J Artif Organs 2004; 27:452-66.

35. Locatelli F, Buoncristiani U, Canaud B, Köhler H, Petitclerc T, Zucchelli P. Dialysis dose and frequency. Nephrol Dial Transplant 2005; 20:285-96.

36. Saran R, Bragg-Gresham JL, Levin NW et al. Longer treatment time and slower ultrafiltration in hemodialysis: associations with reduced mortality in the DOPPS. Kidney Int 2006; 69:1222-8.

37. Marshall MR, Byrne BG, Kerr PG, McDonald SP. Associations of hemodialysis dose and session length with mortality risk in Australian and New Zealand patients. Kidney Int 2006; 69:1229-36.

38. Upadhyay A, Sosa MA, Jaber BL. Single-use versus reusable dialyzers: the known unknowns. Clin J Am Soc Nephrol 2007; 2:1079-86.

39. Lacson E Jr, Lazarus JM. Dialyzer best practice: single use or reuse? Semin Dial 2006; 19:120-8.

40. Twardowski ZJ. Dialyzer reuse-part II: advantages and disadvantages. Semin Dial 2006; 19:217-26. 